\title{
Diffeomorphic Statistical Shape Models
}

\author{
T.F. Cootes, C.J. Twining and C.J. Taylor \\ Imaging Science and Biomedical Engineering, \\ University of Manchester, M13 9PT, UK \\ t.cootes@man.ac.uk
}

\begin{abstract}
We describe a method of constructing parametric statistical models of shape variation which can generate continuous diffeomorphic (non-folding) deformation £elds. Traditional statistical shape models are constructed by analysis of the positions of a set of landmark points. Here we analyse the parameters of continuous warp £elds, constructed by composing simple parametric diffeomorphic warps. The warps are composed in such a way that the deformations are always defned in a reference frame. This allows the parameters controlling the deformations to be meaningfully compared from one example to another. A linear model is learnt to represent the variations in the warp parameters across the training set. This model can then be used to generalise the deformations. Models can be built either from sets of annotated points, or from unlabelled images. In the latter case, we use techniques from non-rigid registration to construct the warp £elds deforming a reference image into each example. We describe the technique in detail and give examples of the resulting models.
\end{abstract}

\section{Introduction}

This paper proposes a method of building generative statistical models of diffeomorphic (ie smooth, invertible) deformation felds. It is part of a larger programme aimed at exploiting the synergy between work on constructing statistical models of shape and that on non-rigid registration.

Statistical models of shape variation [5] have been shown to be powerful tools for image interpretation. Most approaches to constructing such models assume (either explicitly or implicitly) that the object shape can be represented by a set of points which exist on all examples, and essentially de£ne the correspondences across those examples.

The act of annotating a training set with such points is essentially the same as the goal of non-rigid image registration, widely used in the medical image analysis community. In such cases the aim is to £nd the deformation £eld which maps one image into another. Such a £eld de£nes a dense correspondence between two images. Given a set of such dense correspondences, one can build a statistical model of the deformation £eld [11].

When registering two images one usually assumes that similar structures are present in each. Since in general the ordering should not be important, it is desirable that the deformation £eld be smooth and invertible (so that every point in one image has a corre- 
sponding point in the other). Such smooth, invertible transformations are called diffeomorphisms. ${ }^{1}$

In the non-rigid registration literature there are a variety of ways of constructing diffeomorphic maps between pairs of images. The diffeomorphism is either achieved by placing constraints on the Jacobian of more arbitrary warps $[3,11,2]$ or by composing many simple diffeomorphisms [6].

There has been little attention paid to generating parameterisable diffeomorphisms suitable for representing the statistics of shape change across a set of examples.

Point-based statistical models of shape [5] give us a parameterised representation of the movement of a set of landmark points. If we could interpolate the deformation feld between the points we would have a statistical model of continuous deformation. Unfortunately, interpolating in such a way as to ensure the resulting mapping is diffeomorphic is diffcult.

For instance, the simplest approach, that of piecewise linear interpolation using a triangulation of the points, is clearly unsatisfactory. Not only is the resulting deformation feld not smooth, it is quite likely to fold up on itself (see for instance, Figure 1).
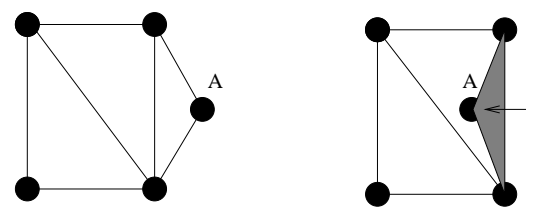

Figure 1: Problems when interpolating with triangles. If point A moves as shown, the triangle xips and there is no longer a 1-1 mapping between the two regions.

Smoother interpolation schemes such as Thin-Plate Splines [1] are also prone to tearing space. However, Twining et al.describe Geodesic Interpolating Splines, which are capable of constructing a diffeomorphism between and two sets of landmark points [13]. Unfortunately the method requires a relatively complex optimisation, so is not very ef£cient for large numbers of points.

Thus a conceptually simple approach, that of using a standard point based statistical model, then interpolating to estimate the diffeomorphism between the mean shape and points generated by the model, can be rather slow.

In this work we describe how we can construct statistical models of continuous deformation felds in such a way that they can only generate smooth invertible mappings. The key to the approach is to create parameterised deformations by composing simple diffeomorphic functions, then to apply statistics in the space of the parameters, rather than to the point positions directly. It is possible to generate functions such that linear interpolation in the parameter space always leads to legal deformations.

In the following we will describe the construction of parameterised diffeomorphic warps, how their parameters can be estimated and how one can model their parameters given a training set of registered data. We will give examples of the models and discuss how such models can be used.

\footnotetext{
${ }^{1}$ In cases where structures appear or disappear between one image and the next, we believe that these should be explicitly modelled as creation or destruction processes - such processes will not be addressed in this paper.
} 


\subsection{Related Work}

Rueckert et al.[11] describe statistical shape models in which deformation is represented by the control points of a B-spline. Since the B-spline is linear in the control point positions, the resulting model is essentially identical to the original PDMs of Cootes et al.[5]. Such a model is prone to generating non-diffeomorphic deformations. However, one could use the constraints on the control point displacements described by Chio and Lee [2] to ensure that B-spline based deformations are diffeomorphic.

The work by Pizer's group on medial representations (M-Reps) [12] explores modelling compound structures and their deformation. In work by Fletcher et al.[8] they investigate statistically modelling the variation in shape in such a way as to preserve correspondence.

\section{Representing Diffeomorphisms}

We can construct complex diffeomorphic functions by composing simple diffeomorphisms. Let $f\left(\mathbf{x} \mid \phi_{i}\right)$ be a diffeomorphic mapping controlled by parameters $\phi_{i}$. We de£ne $f_{1} f_{2}(\mathbf{x})=f_{1}\left(f_{2}(\mathbf{x})\right)$.

We can defne the composition of a set of diffeomorphisms as

$$
F(\mathbf{x} \mid \Phi)=f_{1} f_{2} \ldots f_{n-1} f_{n}(\mathbf{x})
$$

where the parameter vector $\Phi$ is simply a concatenation of the parameter vectors for each individual function, $\left\{\phi_{i}\right\}$. As long as the component functions are all diffeomorphic, so is $F$.

In the following we will consider diffeomorphisms of the form

$$
f_{A} F(\mathbf{x} \mid \Phi)=f_{A} f_{1} f_{2} \ldots f_{n-1} f_{n}(\mathbf{x})
$$

in which $f_{A}(\mathbf{x})$ is an af£ne transformation, and each $f_{i}$ is a parameterised diffeomorphic of increasing complexity and decreasing scale. In particular, we use grid based deformations, in which the deformation of a region is controlled by the position of a set of grid nodes (see Appendix A). We use a coarse grid with few nodes for $f_{1}$, and increase the number of nodes for each subsequent £ner deformation $f_{i}$.

Note that these deformations are similar to the B-spline free form deformations used by Rueckert et al.[11]. However, that work effectively represents the deformation feld with the £nal, single grid based warp, and doesn't necessarily force the result to be diffeomorphic.

The ordering of the transformations is important. To apply a warp to a structure in the reference frame, we frst apply the £nest (small scale) deformations $f_{n}$. We then apply deformations of increasing scale. Finally we apply an af£ne transformation. The grid based warps described in Appendix A are controlled by the position of the nodes. If we apply the deformations in a £ne-to-coarse manner, each grid is always defned in the reference frame, and the displacements of each node can be represented in this frame. This means that it is reasonable to compare the parameters for different deformations, as they are computed in the same domain. It is this property which will allow us to perform meaningful analysis on the parameter vectors when building the models of deformation (see the next section). 
This £ne-to-coarse approach can be considered as applying a series of object-centred deformations. We frst make small changes to the object, then make increasingly large changes, carrying the smaller changes along - see Figure 2. If we applied the coarser warps £rst then the £ner warps would affect different parts of the reference object each time, depending upon which coarse warps are used.

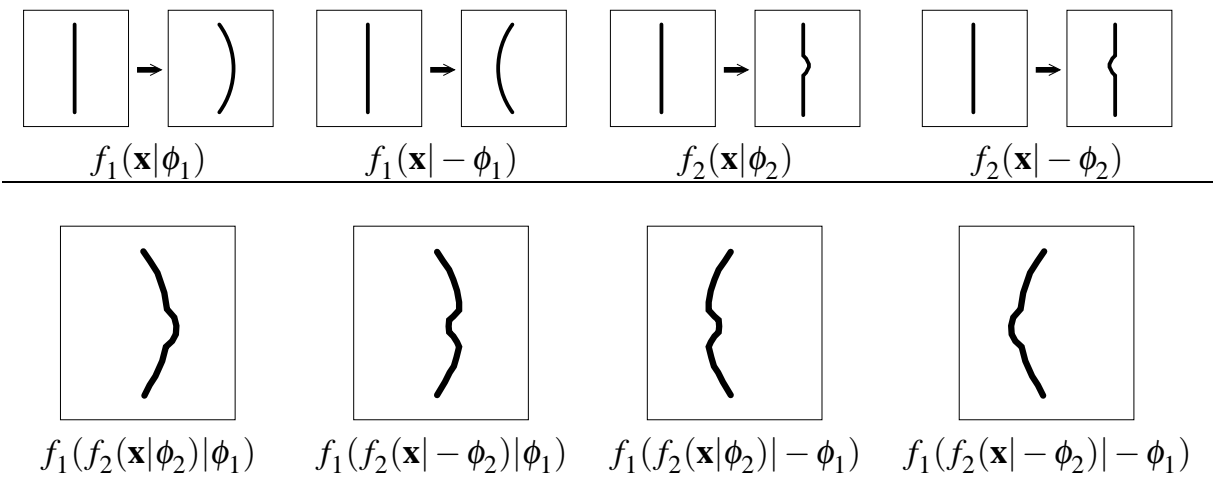

Figure 2: Example of composing warps. $f_{1}$ is a coarse warp, $f_{2}$ a $£$ ne scale warp. By applying $f_{2}$ frst we make small changes to the shape, which are then carried along when we apply $f_{1}$.

\section{Estimating Diffeomorphisms}

We will consider estimating the parameters for the compositional warps described above for two cases; one in which we have a set of landmark positions on a reference shape and a target shape, and one in which we have only un-annotated images (one reference, one target). In both cases we wish to £nd the parameters of the compositional warp, $\phi_{A}$ (the af£ne component) and $\Phi$ (the non-rigid components), which best match the reference to the target data.

\subsection{Estimating from Sets of Points}

Suppose we have a set of points on a reference shape. Assume (for notational convenience) that their co-ordinates are concatenated together into the vector $\mathbf{X}$. Suppose we also have a set of points in the target shape, $\mathbf{X}^{\prime}$. We wish to fnd the parameters which minimise $\left|f_{A} F(\mathbf{X} \mid \Phi)-\mathbf{X}^{\prime}\right|^{2}$.

For small numbers of points there are likely to be multiple possible solutions, as similar deformations can be represented by grids at different scales. We thus tackle the problem in a greedy fashion:

- Find the af£ne parameters $\phi_{A}$ which minimise $\left|f_{A}(\mathbf{X})-\mathbf{X}^{\prime}\right|^{2}$

- For $£$ xed $\phi_{A}$ £nd $\phi_{1}$ to minimise $\left|f_{A} f_{1}(\mathbf{X})-\mathbf{X}^{\prime}\right|^{2}$

- For £xed $\phi_{A}, \phi_{1}$ £nd $\phi_{2}$ to minimise $\left|f_{A} f_{1} f_{2}(\mathbf{X})-\mathbf{X}^{\prime}\right|^{2}$

- Repeat for subsequent functions, £xing previous parameters 


\subsection{Estimating from Images}

Given a pair of unlabelled images, $I_{0}$ and $I_{1}$, we wish to $£$ nd the parameters of the deformation which best matches one onto the other. We will use a modifed version of Rueckert's free-form non-rigid registration algorithm [11]. In the results section below we demonstrate that the compositional technique described here is able to accurately register a pair of images.

Let $\mathbf{X}$ be a set of points densely placed on image $I_{0}$ (for instance, the pixel positions). Let $\mathbf{s}_{0}=I_{0}(\mathbf{X})$ be the intensities sampled at these positions in image $I_{0}$. Let $\mathbf{s}_{1}=I_{1}(W(\mathbf{X}))$ be the intensities sampled at warped positions, $W(\mathbf{X})$ in image $I_{1}$.

We will assume that the quality of match between two images is evaluated using a function $Q\left(\mathbf{s}_{0}, \mathbf{s}_{1}\right)$. In the experiments which follow we use a simple sum of absolute differences, which gives some robustness to outliers. However we could equally use mutual information (MI), normalised MI, or other statistical measures.

The registration process proceeds as follows:

- Find the parameters $\phi_{A}$ of the af£ne transformation which best maps $I_{0}$ onto $I_{1}$ by minimising $Q\left(\mathbf{s}_{0}, I_{1}\left(f_{A}\left(\mathbf{X} \mid \phi_{A}\right)\right)\right)$

- Set the estimate of the deformation to be $F_{0}(\mathbf{X})=f_{A}(\mathbf{X})$.

- Generate a new image, $I_{1}^{(1)}$ by applying the inverse of the transformation $F_{0}(\mathbf{X})$ to $I_{1}$. This can be done by simply writing the samples $\mathbf{s}_{1}=I_{1}\left(F_{0}(\mathbf{X})\right)$ into the grid positions $\mathbf{X}$ in a new image. $I_{1}^{(1)}$ is thus the image $I_{1}$ projected back into the frame of image $I_{0}$

- Find the parameters $\phi_{1}$ of the diffeomorphism $f_{1}\left(\mathbf{X} \mid \phi_{1}\right)$ which best maps $I_{0}$ onto $I_{1}^{(1)}$ by minimising $Q\left(\mathbf{s}_{0}, I_{1}^{(1)}\left(f_{1}\left(\mathbf{X} \mid \phi_{1}\right)\right)\right)$

- Set the estimate of the deformation to be $F_{1}(\mathbf{X})=F_{0} f_{1}(\mathbf{X})$.

- Generate a new image, $I_{1}^{(2)}$ by applying the inverse of the transformation $F_{1}(\mathbf{X})$

- Repeat, optimising each transformation $f_{i}$ in turn, updating $F_{i}=F_{i-1} f_{i}$ and recomputing the back-warped image $I_{1}^{(i+1)}$

The computation of the intermediate images $I_{1}^{(1)}$ is necessary for effciency. For the optimisations with few parameters, we use Nelder-Mead Downhill Simplex [10]. For the larger grid optimisations we estimate the gradient of the objective function by displacing each node in turn, then perform a line search along that direction (the approach used by Rueckert et al.[11]).

The $£$ nal result is the set of all parameters of the warp $F_{n}(\mathbf{X})=f_{A} f_{1} \ldots f_{n}(\mathbf{X})$.

This approach gives us a method of registering a pair of images using a diffeomorphic mapping. In addition, it ensures that since at each stage the warps are de£ned in the reference frame (image $I_{0}$ ), parameters from different warps to different images can be compared. 


\section{Statistical Models of Diffeomorphisms}

Suppose we have a set of training images. If we choose one as a reference, and choose a suitable class of compositional warps (such as the grid-based deformations), we can use the method described above to $£$ nd the diffeomorphic deformation from the reference image into every other image. Each such deformation is summarised by the af£ne parameters, $\phi_{A, i}$ and the parameters controlling the non-rigid warps, concatenated into vectors $\Phi_{i}$.

Shape is usually de£ned as the geometric properties of an object invariant to some transformation (commonly similarity or af£ne). When constructing a statistical shape model from a set of points, Procrustes Analysis [7] is used to remove the effect of such global transformation. In our case the af£ne transformation is encoded in the parameters $\phi_{A}$. Since we are usually only interested in the non-rigid components, we will discard the af£ne terms (note however that in some cases we may wish to retain information about size or skew, in which case we can decompose the af£ne component appropriately).

To build a statistical model, we simply apply Principal Component Analysis (PCA) to the warp parameter vectors $\Phi_{i}$. Where appropriate we should apply a pre-scaling to the elements of each $\Phi_{i}$ so that they are commensurate. This scaling should be chosen so that changing each parameter causes a similar amount of movement of the resulting warp. For instance, with grid based warps, moving one node in a $3 \times 3$ grid by one unit has a four times larger effect than moving one node by the same amount in a 6 x 6 grid. Therefore the parameters controlling the coarser grid should be weighted 4 times more than those of the fner grid.

By selecting a suitable subset of the principal components, we generate a compact linear model with a relatively small number of modes;

$$
\Phi=\hat{\Phi}+\mathbf{P b}
$$

where $\hat{\Phi}$ is the mean of the training set, $\mathbf{P}$ contains the $t$ most signifcant eigenvectors and $\mathbf{b}$ is the vector of shape parameters.

This model can be used to generate new diffeomorphic warps by frst computing the warp parameters, then plugging these into the composition of warps. To ensure that the result is indeed diffeomorphic, we must apply constraints to the parameters $\Phi$. In the case of grid-based warps, this simply involves testing each parameter to ensure it is within a £xed range (see Appendix A). The linear nature of the model ensures that we can use the approach to interpolate between two known diffeomorphic warps by moving along a straight line in $\Phi$-space. This will not generate any illegal parameter values.

The full model of deformation is thus controlled by the global af£ne parameters, $\phi_{A}$ and the model parameters $\mathbf{b}$,

$$
W(\mathbf{x})=f_{A}\left(F(\mathbf{x} \mid \hat{\Phi}+\mathbf{P b}) \mid \phi_{A}\right)
$$

Examples of such models are shown below.

\subsection{Examples from Sets of Points}

Figure 3 shows the $£$ rst three modes of variation of a model trained on deformations estimated from sets of points annotated on a set of face images. A composition of four 
grid-based warps was used, with $2 \times 2,4 \times 4,8 \times 8$ and $16 \times 16$ nodes respectively. 51 faces images taken from the XM2VTS database were used [9]. The nodes at the edges are assumed £xed, so these warps have 2, 18, 98 and 450 degrees of freedom respectively, giving a total of 528. The model explaining $95 \%$ of the variation has 33 degrees of freedom.

Figure 3 shows the continuous deformation of space (using an arbitrary grid) and the effect on the points describing the main features in the reference image.

For comparison Figure 4 shows the $£$ rst 3 modes of a linear statistical shape model trained on the point positions (a PDM) [5] (in this case 21 dimensions are suffcient to capture $95 \%$ of the variance in the training set). The diffeomorphic mode has captured the same sorts of variation.

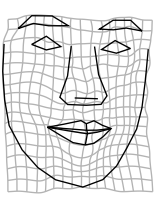

$-3 \sigma_{1}$

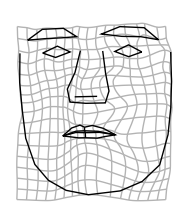

Mode 1
$+3 \sigma_{1}$

1

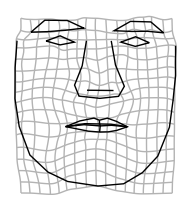

$-3 \sigma_{2}$

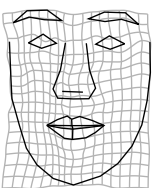

$+3 \sigma_{2}$ Mode 2

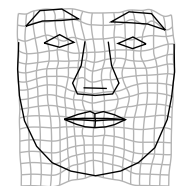

$-3 \sigma_{3}$ Mode 3

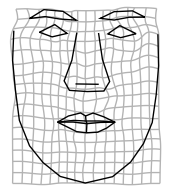

$+3 \sigma_{3}$

Figure 3: Modes of diffeomorphic shape model trained on sets of face points

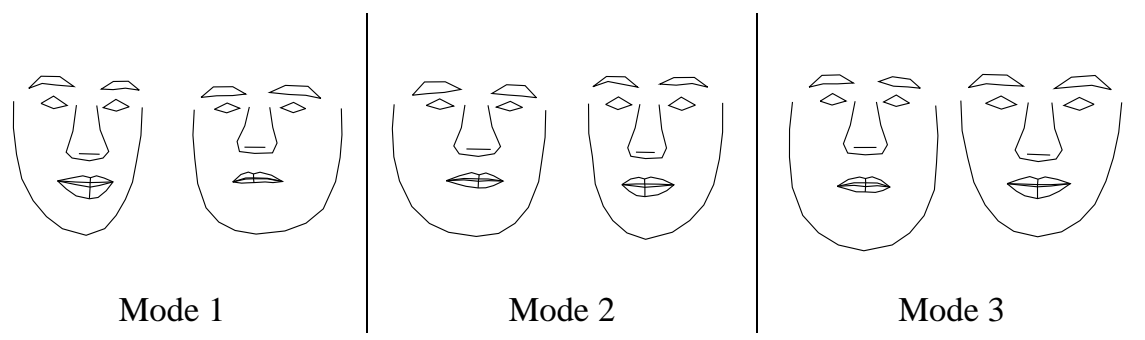

Figure 4: Modes of shape model (PDM) trained on sets of face points

\subsection{Examples from Unlabelled Images}

We used the non-rigid registration technique described in section 3.2 to £nd the warps from the frst face image to subsequent images. We then built a statistical model of the warp parameters. Figure 5 shows the frst three modes of the resulting model of diffeomorphic shape variation. The method has successfully captured similar forms of variation to the models built from annotated landmarks. However, the variation seems a little less 'extreme' (eg around the chin), suggesting that the non-rigid registration has been more conservative when matching.

We can evaluate the quality of the non-rigid registration by projecting the reference points to each target image in turn using the found deformation £eld. These points can then be compared with manual annotations on the same images. We measure the distance from each point to the equivalent curve on the target image, and obtain a mean error of 
1.7 pixels (s.d.0.6 pixels). The faces are about 100 pixels wide. This suggests that the compositional method is giving good results.

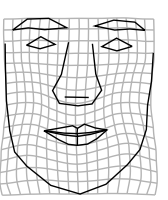

$-3 \sigma_{1}$

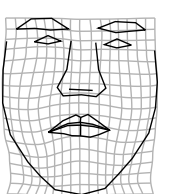

$+3 \sigma_{1}$

Mode 1

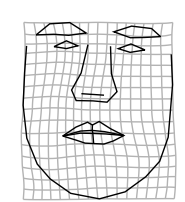

$-3 \sigma_{2}$

Mode 2

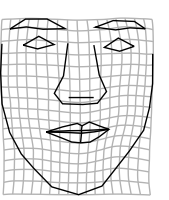

$+3 \sigma_{2}$

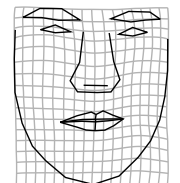

$-3 \sigma_{3}$

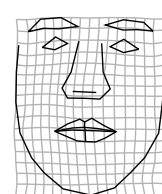

$+3 \sigma_{3}$

Mode 3

Figure 5: Modes of diffeomorphic shape model trained on warps from non-rigid registration of face images

Figure 6 shows the model resulting from applying the technique to a set of $16 \mathrm{MR}$ brain slices. Again, the method has automatically registered the images and found the main modes of variation. Comparing the registration with manual annotation for the central brain structures, we £nd a point to curve error with a mean of 1.7 pixels (s.d. 0.5 pixels). The brains are about 150 pixels across.

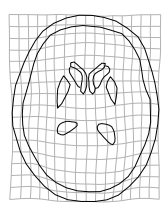

$-3 \sigma_{1}$

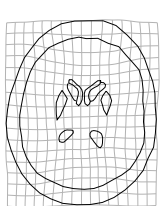

$+3 \sigma_{1}$

Mode 1

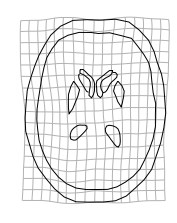

$-3 \sigma_{2}$

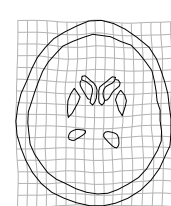

$+3 \sigma_{2}$

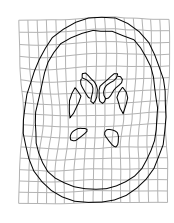

$-3 \sigma_{3}$

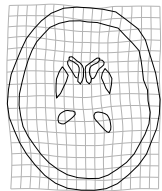

$+3 \sigma_{3}$

Mode 3

Figure 6: Modes of diffeomorphic shape model trained on warps from non-rigid registration of MR brain slice images

\section{Discussion}

The key to the models is the method of generating parameterised classes of diffeomorphisms by composing relatively simple basis functions. Though we have presented results with a particular type of grid-based interpolating warp, a wide range of alternatives could be used. One obvious choice would be the B-splines used to such success by Rueckert $e t$ al.[11], as long as suitable constraints are used to ensure diffeomorphisms [2].

Of course, a difference choice of basis functions will lead to a different model. It is hoped that models built with different but suitably expressive bases will be able to generate similar sorts of deformation £elds, though this remains an area of active investigation.

The grid-based warps limit the sorts of deformation that the method can generate. This means it cannot necessarily get a perfect match to known corresponding points. However, using suf£ciently £ne grids should allow the matching to be 'close enough'.

Though results here are presented for $2 \mathrm{D}$ examples, the extension to $3 \mathrm{D}$ is straightforward. 
The compositional non-rigid registration method described in Section 3.2 was shown to give accurate results, and will be useful in its own right. However, it is a greedy algorithm and potentially prone to local minima. In further work we will investigate its performance more and compare it with alternative registration schemes.

The eventual aim is to use the models in image interpretation. The models appear capable of representing shape variation in a compact form, and should allow fast robust model matching to new images, in the same way that linear models of point position have proved very useful. We are exploring using fast gradient approximation techniques such as that exploited by the Active Appearance Model algorithm [4] for driving the image matching with the models. It is hoped that this will lead to more rapid estimation of deformation between images.

\section{Conclusions}

We have described a method of modelling diffeomorphic shape deformation statistically, leading to a parameterised model capable of synthesising diffeomorphisms ef£ciently. The resulting models have modes of variation similar to those of linear models of point position, but unlike the latter, explicitly de£ne a full diffeomorphic deformation £eld. We anticipate that the models will £nd wide application in the £elds of modelling and interpretting deformable objects, particularly in the medical feld.

\section{Acknowledgements}

The authors would like to thank their colloborators in the MIAS-IRC Project for many fruitful discussions.

\section{A Grid-Based Diffeomorphisms}

We consider diffeomorphic warp £elds represented by deformations of a grid of control points.

The general approach is that the nodes of a regular grid are displaced by some amount, and that displacement is propagated (or interpolated) to estimate the displacement at intermediate positions.

Consider the case in which we are interested in the region $[0, n+1][0, m+1]$, into which we have placed $n \times m$ nodes at integer positions. If the nodes at $(i, j)$ in the reference frame are displaced by $\mathbf{d}_{i j}$, then this implies a deformation of the region as

$$
\begin{aligned}
\mathbf{d}(x, y)= & k(x-i) k(y-j) \mathbf{d}_{i, j} & & +k(i+1-x) k(y-j) \mathbf{d}_{i+1, j} \\
& +k(x-i) k(j+1-y) \mathbf{d}_{i, j+1} & & +k(i+1-x) k(j+1-y) \mathbf{d}_{i+1, j+1}
\end{aligned}
$$

where $i \leq x<i+1$ and $j \leq y<j+1$, and $k(r)$ is a kernel function used to interpolate the deformation. Note that if $k(r)=1-|r|$, then we have bilinear interpolation. However, this is not diffeomorphic since it is not smooth (there are discontinuities in the gradient at integer boundaries).

For a smooth, invertable mapping, it can be shown that $k(r)$ must satisfy

$$
\begin{array}{ccrl}
k(0)=1 & k(-1) & =k(1)=0 \\
k^{\prime}(-1)=k^{\prime}(1)=0 & k(r) & =k(-r)
\end{array}
$$

and that certain limits are placed on the displacements of the nodes.

The simplest polynomial kernel with these properties is $k(r)=\left(1-r^{2}\right)^{2}$. 
An interesting alternative function is

$$
k(r)=0.5(1+\cos (\pi r))
$$

The nice thing about the latter function is that $k(r)+k(1-r)=1$, which means that if four neighbouring nodes are displaced by the same amount then all the points in the enclosed square are translated by that amount, with no other distortions occurring.

By examining the Jacobian of the deformation (5) we can show that the with this trigonometric kernel the deformation is guaranteed to be diffeomorphic if no node is displaced more than $\frac{1}{\pi} \approx$ 0.3183 along $x$ or $y$. Thus we can construct a parameterised diffeomorphism of the form $f(\mathbf{x} \mid \phi)=$ $\mathbf{d}(x, y)$ where the parameters $\phi$ are the $2 n m$ ordinates of the displacements of the nodes. Note that this can easily be extended to $3 \mathrm{D}$ if required.

\section{References}

[1] Fred L. Bookstein. Principal warps: Thin-plate splines and the decomposition of deformations. IEEE Transactions on Pattern Analysis and Machine Intelligence, 11(6):567-585, 1989.

[2] Y. Choi and S. Lee. Injectivity conditions of $2 \mathrm{~d}$ and $3 \mathrm{~d}$ uniform cubic b-spline functions. Graphical Models, 62(6):411-427, 2000.

[3] G. Christensen. Consistent linear-elastic transformations for image matching. In $16^{\text {th }}$ Conference on Information Processing in Medical Imaging, pages 224-237, Visegrád, Hungary, June 1999.

[4] T. F. Cootes, G. J. Edwards, and C. J. Taylor. Active appearance models. In H.Burkhardt and B. Neumann, editors, $5^{\text {th }}$ European Conference on Computer Vision, volume 2, pages 484-498. Springer, Berlin, 1998.

[5] T. F. Cootes, C. J. Taylor, D.H. Cooper, and J. Graham. Active shape models - their training and application. Computer Vision and Image Understanding, 61(1):38-59, January 1995.

[6] T.F. Cootes, S. Marsland, C.J. Twining, K. Smith, and C.J. Taylor. Groupwise diffeomorphic non-rigid registration for automatic model building. In $8^{\text {th }}$ European Conference on Computer Vision, volume 4, pages 316-327. Springer, 2004.

[7] I. Dryden and K. V. Mardia. The Statistical Analysis of Shape. Wiley, London, 1998.

[8] P.T. Fletcher, S. Joshi, C. Lu, and S.M. Pizer. Gaussian distributions on lie groups and their application to statistical shape analysis. In $18^{\text {th }}$ Conference on Information Processing in Medical Imaging, pages 450-462. Springer-Verlag, 2003.

[9] K. Messer, J. Matas, J. Kittler, J. Luettin, and G. Maitre. XM2VTSdb: The extended m2vts database. In Proc. 2nd Conf. on Audio and Video-based Biometric Personal Verifcation. Springer Verlag, 1999.

[10] W.H. Press, S.A. Teukolsky, W.T. Vetterling, and B.P. Flannery. Numerical Recipes in C (2nd Edition). Cambridge University Press, 1992.

[11] D. Rueckert, A.F. Frangi, and J.A. Schnabel. Automatic construction of 3D statistical deformation models using non-rigid registration. In MICCAI, pages 77-84, 2001.

[12] S.M.Pizer, P.T. Fletcher, and S. Joshi ... Deformable m-reps for 3D medical image segmentation. International Journal of Computer Vision, 2-3(55):85-106, 2003.

[13] C. Twining, S. Marsland, and C.J. Taylor. Measuring geodesic distances on the space of bounded diffeomorphisms. In P.L.Rosin and D. Marshall, editors, $13^{\text {th }}$ British Machine Vison Conference, volume 2, pages 847-856. BMVA Press, September 2002. 